\title{
Effects of Multi-Exercise Prescription on Elderly Patients with Mild Cognitive Impairment
}

\author{
Lee-Lan Cheng, Pin-Yu, Wu* \\ Department of Adult and Continuing Education, National Chung-Cheng University, Chiayi, Taiwan, \\ Taiwan, R. O. C. \\ *Corresponding Author: Pin-Yu, Wu, Department of Adult and Continuing Education, National Chung- \\ Cheng University, Chiayi, Taiwan, Taiwan, R. O. C.
}

\begin{abstract}
The purpose of the study is to examine the effects of the intervention of multi-exercise prescription for elderly people with mild cognitive impairment on cognitive function outcomes. The methodology of the study was a pre -post one group experimental design. The pool of available subjects in this study were 25 elderly volunteers with mild cognitive impairment elder (on age 65 or above) who were enrolled in a multiexercise prescription program. For various reasons (illness, 30 percentage absent, not finished test), data were not available for 10 subjects. The final analysis was performed on a sample of 15 volunteer subjects. (age: 78.76 \pm 7.06, height: $150.9 \pm 9.2$, weight: $56.4 \pm 7.24$ ). The material used to measure cognitive function for participations was the Mini Mental State Examination (MMSE). The study was implemented over an eightweek period. Each week was performed $90 \mathrm{~min}$. on a multi-exercise prescription program and twice selfpracticing during the home. A functional physical and mental fitness platform designed and resistance ball were performed on the program. Data analysis were applied to each of the dependent variables. A t-test was use for pre -post one group experimental design. Calculations were made using the Statistical Package for the Social Sciences (SPSS). Result showed that participants demonstrated positive increased cognitive function on three dimensions: total score of MMSE - short-term memory and comprehension. The conclusion indicated that the intervention of multi-exercise prescription is effective method for mild cognitive impairment elder on cognitive function outcomes. The implications for designing multi-exercise prescription within positive increased cognitive function and future research are discussed.
\end{abstract}

Key words: Dementia, Cognitive function, Multi-exercise prescription

\section{INTRODUCTION}

According to active aging proposed by the World Health Organization, participation (the creation of social participation channels), health (the establishment of an environment for physical and mental health), and security (social and economic security and life safety), as well as the comprehensive health promotion concept based on a fitness perspective that was developed by the American College of Sports Medicine (ACSM, 2016). With the increasing number of older adults and life expectancy, the issue of aging population has become more critical, leading to the growing prominence of problems related to elderly health care and treatment (Huang, \& Wang, 2018; Erickson, Weinstein , Lopez, 2012; Wilson , Jackson , Sapey, Lord, 2017). In addition, globally, the prevalence of dementia has been growing rapidly in recent years. These types of well-being complement each other and affect lifestyle and health quality in older adults.

Based on research indicated that substantial progress has been made with respect to recent research on the early symptoms of dementia (Lin, 2011). One characteristic of dementia is an ongoing decline in cognitive function, a process which can last for years. Mild Cognitive Impairment (MCI ) is a transitional stage between normal cognitive function and mild dementia, and it differs from general age-related decline. A loss of memory function in the early stages of the disease gradually develops into the impairment of cognitive functions as a whole, which can occur 6-7 years before the outbreak of dementia. mentioned that Many researches have tried to determine the risk factors for Alzheimer's disease, with hypertension, diabetes, hyperlipemia and obesity, frequent smoking, depression, and a lack of exercise having been identified as the main factors that can cause Alzheimer's disease (Chen, Wang, and Lee, 2016).

Many studies on epidemic diseases conducted during the last 20 years have proposed non- 
pharmacological methods as preventative medicine strategies, including participation in cognitive activities, dietary supplements, and the prescription of exercise or other physical activities (Erickson, Weinstein, \& Lopez, 2012; Voss, Nagamatsu, Liu-Ambrose, \& Kramer, 2011). Many studies have found that physical activity has a positive effect in terms of delaying and preventing cognitive decline and dementia and other cognitive neurological disorders (Kukull, 2006), and physical activity has also been found to improve the physiological mechanisms of newborn nerve cells in the brain and help prevent neuropathological diseases (Huang, Chen, He, \& Huang, 2013). Researchers have also reported that a planned exercise was related to the delay and prevention of dementia and Alzheimer's disease, confirming the value of physical exercise for older adults (Erickson, Weinstein, \& Lopez, 2012; Suzuki, et. al. 2017; Teixeira, et al, 2013). Larson et al. (2006) found that used a self-report evaluation method to calculate the weekly frequency of 15-minute or longer aerobic, stretching, and weight exercises. The results indicated that dementia was present in 13 out of 100065 -year-old and older adults who exercised often, whereas 19.7/1000 older adults who did not exercise often suffered from dementia. Thus, it was inferred that planned exercise was related to the delay and prevention of dementia and Alzheimer's disease, confirming the value of physical exercise for older adults.

Based on the studies, working memory, attention distribution, and the ability to plan are the first functions to deteriorate during MCI. However, not much research has been conducted in the domain of functional fitness training program regarding the prescription of exercise to protect cognitive functions, particularly, memory, attention, and executive control (Wojtek, J., \& Chodzko-Zajko, 2015; Suzuki, et al. 2017). In order to achieve the objective "prevention is better than cure," cognitive functions (memory, attention, and executive control) should be incorporated into the design of exercise prescription courses for older adults, which are believed to be able to postpone the deterioration of cognitive functions in older adults (Lautenschlager, et al, 2008). The purpose of this study was to explore the effects of non-pharmacological interventions, such as physical activities and cognitive training, on the promotion and improvement of cognitive functions in older adults with MCI.

\section{METHOD}

\subsection{Participants}

The participants in this study were adults aged $\geqq 65$ years from Chiayi County community who were diagnosed with MCI by a clinical psychologist. Adults with intellectual inadequacies, physical disabilities, and mental disabilities; adults who were not allowed to exercise according to the instructions of a doctor; and adults with hypertension, past major heart surgery, abnormal chest pain, or sudden dizziness were excluded from the sample. Prior to the experiment, its objectives, content, measurement items, and matters requiring attention were explained to the participants, who signed consent forms confirming their voluntary participation in this study.

\subsection{Intervention Materials}

\section{$>$ Multi-exercise Course Design:}

The design of the multi-exercise course in this study was developed with reference to the standards established by the American College of Sports Medicine (ACSM). Following the ACSM's (2015) exercise recommendations and Miyake and Yamazaki's healthy activity design for older adults, the multi-exercise group training course integrated working memory, attention distribution, and the ability to plan in order to improve the participants' cognitive functions. Reference factors of course design included course schedule and form, course content and auxiliary tools, course planning constructs, movement selection principles, and the design of training elements promoting cognitive function. The multi-exercise course design was tested for expert validity by fitness experts and clinical psychologists.

\section{$>$ Instrument of Intervention:}

The design of the intelligent board hardware, a group exercise intervention tool, included a $4 \mathrm{M}^{*} 1.5 \mathrm{M}$ artificial grass field for physical activities. The various shapes, colors, lines, and numbers on the surface corresponded to cognitive science functions and conformed with requirements regarding older adults' safety, tactile sensations, interactions, and ergonomics (Huang, Wang, \& Lin, 2016). The course design focused on the training of cognitive functions using an intelligent board and exercise 
ball, and aimed to improve cognitive performance in older adults with MCI. The duration of the intervention process was 90 minutes, which included 15 minutes of dynamic aerobic warm-up exercises, 30 minutes of resistance training, 30 minutes of cognitive function training, and 15 minutes of stretching.

Posttest.

The cognitive functions of the participants were evaluated using the MMSE. The Chinese and English versions of the MMSE are similar (Fu, 2008). The MMSE has been a common tool used for the clinical evaluation of cognitive functions since 1975 and is one of the most widely used cognitive examination tools worldwide (Folstein, Folstein, \& McHugh, 1975). The MMSE is also recommended by the National Institute for Health and Clinical Excellence (NICE) as a standard tool for dementia screening. The MMSE score indicates the severity of dementia, and the maximum score is 30 points. The scale consists of 11 items and 30 answers, with 1 point given for each correct answer. A score of 24-30 points indicates intact cognitive functions; a score of 18-23 points indicates MCI; a score of 017 points indicates severe cognitive impairment. The 11 items of the scale include five constructs, namely, orientation, registration, attention and calculation, recall, and language. Their content examines conceptions of current time and place, the repetition of words, simple mathematical calculations, and language use and understanding (Folstein et al., 1975).

\subsection{Procedures}

Prior to the experiment, the Mini-Mental State Examination (MMSE) was administered to each participant by a clinical psychologist to evaluate participant's cognitive functions. The exercise course was conducted by a trained teacher. The participants completed a pre-test that covered their basic personal data, health status, and cognitive function status one week before the exercise course intervention. Any participant who expressed an unwillingness to continue to participate, or experienced physical discomfort, dizziness, difficult breathing, paleness, chest pain, or fatigue during the 8-week exercise intervention immediately stopped exercising. Each week was performed $90 \mathrm{~min}$. on a multi-exercise prescription program and twice self-practicing during the home.

The pretest was administered during the first week. The course design focused on the training of cognitive functions using an intelligent board and exercise ball, and aimed to improve cognitive performance in older adults with MCI. The duration of the intervention process was 90 minutes, which included 15 minutes of dynamic aerobic warm-up exercises, 30 minutes of resistance training, 30 minutes of cognitive function training, and 15 minutes of stretching. A Content of the cognitive function training course during Week 1-2 (Working memory ); . Week 3-5 (Attention distribution); Week 6-8 (Ability to plan). Upon completion the eighth week of the lesson, each participant individually completed the final week of the study.

\subsection{Design and Data Analysis}

This study applied a pre-experimental design and conducted a one-group pretest-posttest experiment. The experimental group consisted of older adults with MCI. A multi-exercise course intervention was conducted over eight weeks and included one 90-minute group intervention by an instructor and two home-based exercise sessions per week. Participants were provided with reference pictures to guide them in performing the home exercises.

The statistical analyses were conducted using SPSS for Windows 22.0 for the data collected in this study: Basic data was analyzed using descriptive statistics to explore the distribution of basic variables among the participants. The t-test was used to analyze MMSE scores before and after the course intervention; $\alpha=.05$ indicated statistical significance.

\section{RESUlTS}

\subsection{Participants' Basic Data}

This study recruited 25 participants. Older adults who did not have MCI, had an attendance rate that was lower than $70 \%$, or did not complete the entire test were excluded from the sample after the eightweek multi-exercise training course. The final sample included 15 participants. Table 1 shows the results of the basic data survey. With regard to gender, most (66\%) of the participants were female, while two participants 33\%) were male. The average age was $77.76 \pm 7.06$ years. The average height was $155.9 \pm 9.2 \mathrm{~cm}$. The average weight was $55.4 \pm 7.24 \mathrm{~kg}$. Basic data showed in Table 1 . 
Table1. Participants' basic data survey

\begin{tabular}{|c|c|c|c|c|}
\hline Gender & \multicolumn{2}{|c|}{ Number } & \multicolumn{2}{c|}{ Percentage } \\
\hline Male & \multicolumn{2}{|c|}{$53 \%$} \\
\hline Female & \multicolumn{2}{|c|}{10} & Max. & Min. \\
\hline Variable & Mean & SD & 63 & 93 \\
\hline Age (years) & 77.76 & 7.06 & 179 & 141 \\
\hline Height (cm) & 155.9 & 9.2 & 46 & 68.2 \\
\hline Weight (kg) & 55.4 & 7.24 & \\
\hline
\end{tabular}

\subsection{Effects of Cognitive Functions}

The results in Table 2 showed that in time orientation, spatial orientation, three-character word registration, attention and calculation, short-term memory, ability to recall names, repetition, understanding, reading, writing, drawing, and total MMSE scores after the multi-exercise course intervention.

1) With regard to time orientation, its values were $2.82 \pm 1.561$ and $3.55 \pm 1.553$ before and after the intervention, respectively. The average values of the time orientation sense were 2.80 and 3.53 before and after the intervention, respectively. However, the before and after results were not significantly different $(p=.052, p>0.5)$.

2) The value for spatial orientation was $4.75 \pm .799$ before the intervention and $5 \pm .000$ after the intervention. Its average values were 4.75 and 5.00 before and after the intervention, respectively. However, the before and after results were not significantly different $(p=.219, p>0.5)$.

3) With regard to the repetition of three-character words, the value was $2.89 \pm .352$ both before and after the intervention, and the average value did not change. The before and after results were not significantly different $(p=1, p>0.5)$.

4) The attention and calculation score was $.95 \pm 1.534$ points before the intervention and $1.55 \pm 1.959$ points after the intervention. The average scores were .93 and 1.53 before and after the intervention, respectively. However, the before and after results were not significantly different $(p=.228, p>0.5)$.

5) The short-term memory score was $.42 \pm .737$ before the intervention and $2.33 \pm 1.113$ after the intervention. The average scores were .42 and 2.35 before and after the intervention, respectively, reaching a significant difference $(p=.000, p<0.5)$.

6) With regard to understanding, the score both before and after the intervention was $.29 \pm .458$. The average score increased from 1.69 before the intervention to 2.80 after the intervention, reaching a significant difference $(p=.002, p<0.5)$.

7) The reading score both before and after the intervention was $2.89 \pm .352$, and the average reading score did not change. The before and after results were not significantly different ( $p=1, p>0.5)$.

8) The writing score was $.09 \pm .258$ before the intervention and $.29 \pm .458$ after the intervention. The average score increased from .07 before the intervention to .27 after the intervention. However, the before and after results were not significantly different ( $p=.082, p>0.5)$.

9) The drawing score before and after the intervention was $.35 \pm .488$ and $.49 \pm .516$, respectively. The average score increased from .33 before the intervention to .47 after the intervention. However, the before and after results were not significantly different $(p=.546, p>0.5)$.

10) The total MMSE score was $17.09 \pm 3.127$ before the intervention and $21.95 \pm 3.845$ after the intervention. The average score increased from 17.07 before the intervention to 21.93 after the intervention, reaching a significant difference $(p=.001, p<.05)$.

11) The average scores for the ability to recall names and repetition did not change, and thus did not reach a statistically significant difference.

The statistical results indicated a significant change in the participants' MMSE total scores, short-term 
memory, and understanding, as well as increases in their scores for time orientation, spatial orientation, attention and calculation, writing, and drawing, meaning that the participants' cognitive functions had improved after the training course intervention.

Table2. Changes in MMSE scores

\begin{tabular}{|l|l|l|l|l|}
\hline Variable & $\begin{array}{l}\text { Before } \\
\text { Intervention }\end{array}$ & After Intervention & t-value & $p$-value \\
\hline Time orientation & $2.82 \pm 1.568$ & $3.53 \pm 1.552$ & -2.128 & .052 \\
\hline Spatial orientation & $4.75 \pm .799$ & $5.00 \pm .000$ & -1.293 & .217 \\
\hline $\begin{array}{l}\text { Three-character word } \\
\text { registration }\end{array}$ & $2.89 \pm .352$ & $2.89 \pm .352$ & .000 & 1 \\
\hline $\begin{array}{l}\text { Attention and } \\
\text { calculation }\end{array}$ & $.95 \pm 1.534$ & $1.55 \pm 1.959$ & -1.260 & .228 \\
\hline Short-term memory & $.42 \pm .737$ & $2.35 \pm 1.113$ & -6.808 & $.000^{* * *}$ \\
\hline Understanding & $1.69 \pm .976$ & $2.82 \pm .414$ & -3.900 & $.002^{* *}$ \\
\hline Reading & $.29 \pm .458$ & $.29 \pm .458$ & .000 & 1 \\
\hline Writing & $.09 \pm .258$ & $.29 \pm .458$ & -1.871 & .082 \\
\hline Drawing & $.35 \pm .488$ & $.49 \pm .516$ & -.619 & .546 \\
\hline Total & $17.09 \pm 3.127$ & $21.95 \pm 3.845$ & -4.172 & $.001^{* *}$ \\
\hline
\end{tabular}

\section{DisCUSSION}

The purpose of the study is to examine the effects of the intervention of multi-exercise prescription for elderly people with mild cognitive impairment on cognitive function outcomes. The results in this study indicated significant impacts of the multi-exercise intervention on MMSE total scores, shortterm memory, and understanding in older adults with MCI. Positive progress was observed in terms of time orientation, spatial orientation, attention and calculation, writing, and drawing. These results indicated the positive effects of the training course on different cognitive functions. The results confirmed the ability of stimulation to promote the cognitive functions that are the earliest to deteriorate (working memory, attention distribution, and the ability to plan) in older adults with MCI, thus supporting the proposition regarding healthy activity design that was developed by Miyake and Yamazaki (Lin \& Lee, 2013). The multi-exercise training course designed in this study was shown to have improved cognitive functions in older adults with MCI.

The multi-exercise training course designed is an essential construct for cognitive and functional fitness in many theories of learning and instruction. However, while the benefits of exercise program in general might be taken for granted, uncertainty still exists as to how to select and optimize uses of different exercise prescription depending on myriad psychosocial characteristics of frail adults and instructional module or prescription.

This study has some implications for those who design instruction. Instructional functional fitness program can successfully increase effects of participant with MCI. Future studies should also examine other client characteristics that might influence outcomes in instructional settings. The relatively short duration of the treatment and the effect of instructional control may have influenced the outcomes. Extending the experimental time for overall instruction could produce different results for performance. Further research should identify critical elements on multi-exercise training course under which effective interaction can be generated and maintained on the domain of training setting for older adults. with MCI. More research should examine the effects of varieties of instructional strategies in varied new and emerging technology during training environments.

\section{REFERENCES}

[1] American College of Sports Medicine. (2016). ACSM'S exercise for older adults. Philadelphia, USA.

[2] American College of Sports Medicine. (2015). Exercise and physical activity for older adults. Medicine and Science in Sports and Exercise, 41, 1510-1530.

[3] Chen, C. M., Wang, H. H., \& Lee, S. F. (2016). Exercise prescription for patients with Alzheimer's disease. Annual Journal of Physical Education and Sports Science, (137), 37-44.

[4] Chu, Y. H. (2013). The effects of physical activity in cognitive function. The Journal of Long-term Care, 17(1), 11-21

[5] Erickson, K. I, Weinstein AM, Lopez O. L. (2012). Physical activity, brain plasticity, and Alzheimer's Disease. Arch Med., Res; 43:615-21. 
[6] Folstein, M. F., Folstein, S. E., \& McHugh, P. R. (1975). "Mini-mental state" : a practical method for grading the cognitive state of patients for the clinician. Journal of psychiatric research, 12(3), 189-198.

[7] Huang, C.Y., Wang, H. H. \& Lin, M. J. (2016) . Annual GlobalReport on Innovatuion in Active, Healthy and Smart Aging Sector. The Second International Forum on Senior Care Science and Innovation. 26th28th October, 2016, in Beijimg, China.ISBN:978-7-5304-8479-1.

[8] Huang, C. Y., \& Wang, H. H. (2018). Prescribing Exercise for Frail Elders: A Cognicise Fitness Training Intervention as an Example. Annual global report on Innovation in Active, Healthy and Smart Ageing Sector. Beijing Science \& Technology Publishing Press.

[9] Kukull, W. (2006). Exercise is associated with reduced risk for incident dementia among persons 65 years of age and older. Annals of internal medicine, 144(2), 73-81. doi: 10.7326/0003-4819-144-2-20060117000004Suzuki, T., , Shimada, H., Makizako, H., Doi, T., Yoshida, D., Tsutsumimoto, K., Anan, Y., Uemura, K., Lee, S., and Park, H. (2017). Community-Based Intervention for Prevention of Dementia in Japan, The Journal of Prevention of Alzheimer's Disease - JPAD. Volume 2, Number ,1-6.

[10] Larson, E. B., Wang, L., Bowen, J. D., McCormick, W. C., Teri, L., Crane, P., \& Liu-Ambrose, T., \& Donaldson, M. G. (2009) . Exercise and cognition in older adults: is there a role for resistance training programmes? British Journal of Sports Medicine, 43(1), 25-27. doi:10.1136/bjsm.2008.055616.

[11] Lautenschlager, N. T., Cox, K. L., Flicker, L., Foster, J. K., van Bockxmeer, F. M., Xiao, J., ... \& Almeida, O. P. (2008) . Effect of physical activity on cognitive function in older adults at risk for Alzheimer disease: a randomized trial. Jama, 300(9), 1027-1037. doi:10.1001/jama.300.9.1027Lin, C. C. (2011). On mild cognitive impairment. Retrieved from: https://www1.cgmh.org.tw/dementiahel/DementiaInformation1 1.html

[12] Teixeira, C. V. L., Gobbi, S., Pereira, J. R., Vital, T. M., Hernandéz, S. S., Shigematsu, R., \& Gobbi, L. T. B. (2013) . Effects of square-stepping exercise on cognitive functions of older people. Psychogeriatrics, 13(3), 148-156. doi:10.1111/psyg.12017Wang, C. H., \& Tsai, C. L. (2011). The benefits of exercise on brain function: A review. Research in Applied Psychology, (50), 191-216.

[13] Voss, M. W., Nagamatsu, L. S., Liu-Ambrose, T., \& Kramer, A. F. (2011) . Exercise, brain, and cognition across the life span. Journal of applied physiology, 111(5), 1505-1513. doi: 10.1152/japplphysiol.00210.2011

[14] Wilson D, Jackson T, Sapey E, Lord J. M. ( 2017). Frailty and sarcopenia: The potential role of an aged immune system. Ageing Res Rev. Jul;36:1-10.

[15] Wojtek, J., \& Chodzko-Zajko, (2015). ACSM's Exercise for older adults. Wolters Kluwer Health, Philadelphia.

\section{AUTHORS' BIOGRAPHY}

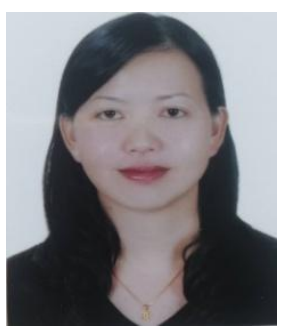

Lee-Lan Cheng, is a candidate student of $\mathrm{Ph}$. D program in Department of Adult and Continuing Education, National Chung-Cheng University, Chiayi, Taiwan, Taiwan, R. O. C. Currently, teaches physical education and functional fitness for older adults in society and is also an external evaluator for the fitness and consultant at a fitness promotion for society senior citizens. Cheng's field of expertise lies in sports and health promotion, sports pedagogy, Physical activity instruction of older adults, and the design of educational sports programs for active-aging learners.

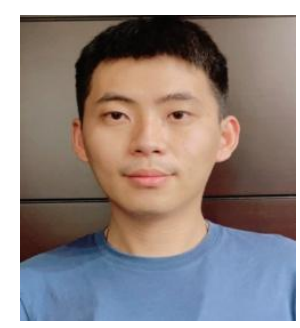

Pin-YuWu, is a candidate student of Ph. D program in Department of Adult and Continuing Education, National Chung-Cheng University, Chiayi, Taiwan, Taiwan, R. O. C.Wu's field of expertise lies in sports, health and recreation management, physical activity instruction of older adults, and the design of sport management for active-aging learners.

Citation: Lee-Lan Cheng, Pin-Yu, Wu. "Effects of Multi-Exercise Prescription on Elderly Patients with Mild Cognitive Impairment" International Journal of Humanities Social Sciences and Education (IJHSSE), vol 8, no. 5, 2021, pp. 35-40. doi: https://doi.org/10.20431/2349-0381.0805005.

Copyright: (C) 2021 Authors. This is an open-access article distributed under the terms of the Creative Commons Attribution License, which permits unrestricted use, distribution, and reproduction in any medium, provided the original author and source are credited. 